Mathematical Modelling and Analysis

Volume 18 Number 1, February 2013, 136-148

http://dx.doi.org/10.3846/13926292.2013.760114

(c) Vilnius Gediminas Technical University, 2013
Publisher: Taylor\&Francis and VGTU

http://www.tandfonline.com/TMMA

Print ISSN: 1392-6292

Online ISSN: 1648-3510

\title{
A Central Part Interpolation Scheme for Log-Singular Integral Equations
}

\section{Kerli Orav-Puurand}

\section{University of Tartu}

J. Liivi 2, 50409 Tartu, Estonia

E-mail: puurand@ut.ee

Received March 26, 2012; revised October 12, 2012; published online February 1, 2013

\begin{abstract}
A fully discrete high order method is constructed and justified for a class of Fredholm integral equations of the second kind with kernels that may have boundary and logarithmic diagonal singularities. The method is based on the improving the boundary behaviour of the kernel with the help of a change of variables, and on "central part" interpolation by polynomials on the uniform grid.
\end{abstract}

Keywords: weakly singular integral equations, logarithmic diagonal singularity, collocation method, product integration, central part interpolation.

AMS Subject Classification: 65R20; 65D05; 65D07; 65D30.

\section{Introduction}

In the present paper we treat a fully discrete method of accuracy order $O\left(h^{m}\right)$ for the integral equation

$$
u(x)=\int_{0}^{1}[a(x, y) \log |x-y|+b(x, y)] u(y) d y+f(x), \quad 0 \leq x \leq 1,
$$

with the logarithmic diagonal singularity in the kernel. The coefficient functions $a, b \in C^{m}([0,1] \times(0,1))$ and the free term $f \in C[0,1] \cap C^{m}(0,1)$ may have certain boundary singularities characterised in Lemma 1 and Lemma 2 below. The method is based on a smoothing change of variables (see for example $[3,4,6,7,10])$ and a product integration method based on the "central part" interpolation on the uniform grid, which has also been used in [8] for solving weakly singular integral equation. We rely on the fact that the "central part" interpolation of smooth functions by polynomials and by high order polynomial splines on uniform grids has excellent accuracy and stability properties comparable with the accuracy and stability properties of Chebyshev interpolation [16]. The use of data only on uniform grids is preferable also from the algorithmical point of view due to better numerical stability. See Section 3 for the details about the "central part" interpolation. In Section 2 we reduce problem 
(1.1) by the smoothing change of variables to the form with smooth data and solution.

The present paper is a complement to work [14], where the convergence and the error of the product quasi-interpolation method has been presented for the integral equation (1.1). We refer yet to [9], where another type fully discrete method for solving (1.1) has been constructed.

Denote by $T$ the integral operator of equation (1.1)

$$
(T u)(x)=\int_{0}^{1}[a(x, y) \log |x-y|+b(x, y)] u(y) d y .
$$

For $m \in \mathbf{N}, \lambda_{0}, \lambda_{1}<1$, denote by $C^{m, \lambda_{0}, \lambda_{1}}(0,1)$ the weighted space of functions $u \in C[0,1] \cap C^{m}(0,1)$ such that,

$$
\|u\|_{C^{m, \lambda_{0}, \lambda_{1}(0,1)}}:=\sum_{k=0}^{m} \sup _{0<x<1} \omega_{k-1+\lambda_{0}}(x) \omega_{k-1+\lambda_{1}}(1-x)\left|u^{(k)}(x)\right|<\infty,
$$

where

$$
\omega_{\rho}(r)= \begin{cases}1, & \rho<0, \\ r^{\rho} /(1+|\log r|), & \rho \geq 0, \quad r, \rho \in \mathbf{R}, r>0 .\end{cases}
$$

Clearly, $C^{m}[0,1] \subset C^{m, \lambda_{0}, \lambda_{1}}(0,1)$ for $\lambda_{0}, \lambda_{1}<1$.

Lemma 1. Let $a, b \in C([0,1] \times(0,1))$ satisfy for $(x, y) \in[0,1] \times(0,1)$ the inequality

$$
|a(x, y)|+|b(x, y)| \leq c y^{-\lambda_{0}}(1-y)^{-\lambda_{1}},
$$

where $\lambda_{0}, \lambda_{1} \in \mathbf{R}, \lambda_{0}<1, \lambda_{1}<1$. Then $T$ maps $C[0,1]$ into $C[0,1]$, and $T: C[0,1] \rightarrow C[0,1]$ is compact.

The proof is standard, cf. [5]; a detailed argument can be found in [11].

Denote $\partial_{x}^{k} \partial_{y}^{l}=(\partial / \partial x)^{k}(\partial / \partial y)^{l}$.

Lemma 2 [see [11]]. Let $a, b \in C^{m}([0,1] \times(0,1))$ and let for $k+l \leq m$, $(x, y) \in[0,1] \times(0,1)$,

$$
\left|\partial_{x}^{k} \partial_{y}^{l} a(x, y)\right|+\left|\partial_{x}^{k} \partial_{y}^{l} b(x, y)\right| \leq c y^{-\lambda_{0}-l}(1-y)^{-\lambda_{1}-l},
$$

where $\lambda_{0}<1, \lambda_{1}<1$. Then $T$ maps the Banach space $C^{m, \lambda_{0}, \lambda_{1}}(0,1)$ into itself, and $T: C^{m, \lambda_{0}, \lambda_{1}}(0,1) \rightarrow C^{m, \lambda_{0}, \lambda_{1}}(0,1)$ is compact.

Let $I$ be the identity mapping and denote $\mathcal{N}(I-T)=\{u \in C[0,1]: u=T u\}$. The following theorem is a consequence of Lemmas 1 and 2 .

Theorem 1. Assume the conditions of Lemma 2 and $\mathcal{N}(I-T)=\{0\}$. Then for $f \in C^{m, \lambda_{0}, \lambda_{1}}(0,1)$ also the solution of equation $(1.1) u \in C^{m, \lambda_{0}, \lambda_{1}}(0,1)$ and $\|u\|_{C^{m, \lambda_{0}, \lambda_{1}(0,1)}} \leq c\|f\|_{C^{m, \lambda_{0}, \lambda_{1}(0,1)}}$. The constant $c$ is independent of $f$.

Our main results will be established under assumptions of Theorem 1. 


\section{The Smoothing Change of Variables}

In the integral equation (1.1) we perform the change of variables

$$
x=\varphi(t), \quad y=\varphi(s), \quad 0 \leq t \leq 1,0 \leq s \leq 1,
$$

where $\varphi:[0,1] \rightarrow[0,1]$ is defined by the formula

$$
\begin{gathered}
\varphi(t)=\frac{1}{c_{*}} \int_{0}^{t} \sigma^{p_{0}-1}(1-\sigma)^{p_{1}-1} d \sigma \\
c_{*}=\int_{0}^{1} \sigma^{p_{0}-1}(1-\sigma)^{p_{1}-1} d \sigma=\frac{\left(p_{1}-1\right) !}{p_{0}\left(p_{0}+1\right) \cdots\left(p_{0}+p_{1}-1\right)} .
\end{gathered}
$$

We assume, that $p_{0}, p_{1} \in \mathbf{N}$. If so, the integral in (2.1) can be evaluated in a stable way by an exact Gauss rule, since the integrand is a polynomial of degree $p_{0}+p_{1}-2$. Clearly, $\varphi(0)=0, \varphi(1)=1$ and $\varphi(t)$ is strictly increasing in $[0,1]$. Hence $\frac{\varphi(t)-\varphi(s)}{t-s}>0,|\varphi(t)-\varphi(s)|=\frac{\varphi(t)-\varphi(s)}{t-s}|t-s|$ for $s \neq t$, and equation (1.1) takes the form

$$
v(t)=\int_{0}^{1}(A(t, s) \log |t-s|+B(t, s)) v(s) d s+g(t), \quad 0 \leq t \leq 1,
$$

where $v(t)=u(\varphi(t))$ is the new function we look for,

$$
\begin{aligned}
& g(t)=f(\varphi(t)), \quad A(t, s)=a(\varphi(t), \varphi(s)) \varphi^{\prime}(s), \\
& B(t, s)=[a(\varphi(t), \varphi(s)) \log \Phi(t, s)+b(\varphi(t), \varphi(s))] \varphi^{\prime}(s), \\
& \Phi(t, s)=\left\{\begin{array}{ll}
(\varphi(t)-\varphi(s)) /(t-s), & t \neq s \\
\varphi^{\prime}(s), & t=s
\end{array}\right\} .
\end{aligned}
$$

Let us characterise the boundary behaviour of functions in equation (2.2). Clearly,

$$
\begin{aligned}
& 0 \leq \varphi(t) \leq c t^{p_{0}}, \quad 0 \leq 1-\varphi(t) \leq c(1-t)^{p_{1}} \\
& \left|\varphi^{(k)}(t)\right| \leq c t^{p_{0}-k}(1-t)^{p_{1}-k}, \quad 0<t<1, k=1, \ldots, m .
\end{aligned}
$$

Lemma 3. Let $a$ and $b$ satisfy the conditions of Lemma 2. Then for $j=$ $0, \ldots, m, 0 \leq t \leq 1,0<s<1$, it holds

$$
\left|\partial_{s}^{j} a(\varphi(t), \varphi(s))\right|+\left|\partial_{s}^{j} b(\varphi(t), \varphi(s))\right| \leq c s^{-p_{0} \lambda_{0}-j}(1-s)^{-p_{1} \lambda_{1}-j} .
$$

The proof of the inequality is based on the formula of Faà di Bruno

$$
\begin{aligned}
& \left(\frac{d}{d x}\right)^{j} u(\varphi(x)) \\
& \quad=\sum_{k_{1}+2 k_{2}+\cdots+j k_{j}=j} \frac{j !}{k_{1} ! \cdots k_{j} !} u^{\left(k_{1}+\cdots+k_{j}\right)}(\varphi(x))\left(\frac{\varphi^{\prime}(x)}{1 !}\right)^{k_{1}} \cdots\left(\frac{\varphi^{(j)}(x)}{j !}\right)^{k_{j}}
\end{aligned}
$$


where the sum is taken over all non-negative integers $k_{1}, \ldots, k_{j}$ such that $k_{1}+$ $2 k_{2}+\cdots+j k_{j}=j$.

The derivatives of the function $\Phi(t, s)$ have singularities at $(0,0)$ and $(1,1)$, the only zeroes of $\Phi(t, s)$ in $[0,1] \times[0,1]$. It is easy to see that

$$
\partial_{s}^{k} \Phi(t, s) \asymp(t+s)^{p_{0}-k-1}((1-t)+(1-s))^{p_{1}-k-1} \text {, as } t, s \rightarrow 0 \text { or as } t, s \rightarrow 1,
$$

that together with the formula of Faà di Bruno implies the following results.

Lemma 4. For $j=0, \ldots, m, 0 \leq t \leq 1,0<s<1$, it holds

$$
\left|\partial_{s}^{j}(\log (\Phi(t, s)))\right| \leq c(t+s)^{-j}((1-t)+(1-s))^{-j}
$$

Next we present estimates for functions $A(t, s), B(t, s)$, and $\partial_{s}^{m}[A(t, s) v(s)]$, $\partial_{s}^{m}[B(t, s) v(s)]$.

Lemma 5. Let $a$ and $b$ satisfy the conditions of Lemma 1. Then the following holds true: if $p_{0}, p_{1} \geq 1$ satisfy

$$
p_{0}>1 /\left(1-\lambda_{0}\right), \quad p_{1}>1 /\left(1-\lambda_{1}\right),
$$

then for $(t, s) \in[0,1] \times(0,1)$ it holds

$$
|A(t, s)| \leq c s^{\delta_{0}}(1-s)^{\delta_{1}}, \quad|B(t, s)| \leq c s^{\delta_{0}}(1-s)^{\delta_{1}}|\log s(1-s)|,
$$

with $\delta_{0}:=\left(1-\lambda_{0}\right) p_{0}-1>0, \delta_{1}:=\left(1-\lambda_{1}\right) p_{1}-1>0$.

Lemma 6 [see [13]]. Let the conditions of Lemma 2 be fulfilled. If $p_{0}, p_{1} \geq 1$ satisfy

$$
p_{0}>m /\left(1-\lambda_{0}\right), \quad p_{1}>m /\left(1-\lambda_{1}\right),
$$

then for $(t, s) \in[0,1] \times(0,1)$ it holds

$$
\begin{aligned}
& |A(t, s)| \leq c s^{m-1+\delta_{0}}(1-s)^{m-1+\delta_{1}}, \\
& |B(t, s)| \leq c s^{m-1+\delta_{0}}(1-s)^{m-1+\delta_{1}}|\log s(1-s)|,
\end{aligned}
$$

with $\delta_{0}:=\left(1-\lambda_{0}\right) p_{0}-m>0, \delta_{1}:=\left(1-\lambda_{1}\right) p_{1}-m>0$. About the boundary behaviour of $v(t)=u(\varphi(t))$ see Lemma 3.1 in [13]: for $u \in C^{m, \lambda_{0}, \lambda_{1}}(0,1)$, $j=1, \ldots, m, 0<t<1$, it holds

$$
\begin{aligned}
& \left|v^{(j)}(t)\right| \leq c\|u\|_{C^{m, \lambda_{0}, \lambda_{1}(0,1)}}\left\{\begin{array}{ll}
t^{p_{0}-j}, & \lambda_{0}<0 \\
t^{\left(1-\lambda_{0}\right) p_{0}-j}|\log t|, & 0 \leq \lambda_{0}<1
\end{array}\right\} \\
& \quad \times\left\{\begin{array}{l}
(1-t)^{p_{1}-j}, \\
\lambda_{1}<0 \\
(1-t)^{\left(1-\lambda_{1}\right) p_{1}-j}|\log (1-t)|, \quad 0 \leq \lambda_{1}<1
\end{array}\right\}, \\
& \left|\partial_{s}^{m}[A(t, s) v(s)]\right| \leq c s^{-1+\delta_{0}}(1-s)^{-1+\delta_{1}}\|u\|_{C^{m, \lambda_{0}, \lambda_{1}}(0,1)}, \\
& \left|\partial_{s}^{m}[B(t, s) v(s)]\right| \leq c s^{-1+\delta_{0}}(1-s)^{-1+\delta_{1}}|\log s(1-s)|\|u\|_{C^{m, \lambda_{0}, \lambda_{1}(0,1)}} .
\end{aligned}
$$

We see, that under conditions (2.5) and $0 \leq \lambda_{0}<1,0 \leq \lambda_{1}<1$, it holds

$$
v^{(j)}(0)=v^{(j)}(1)=0, \quad j=1, \ldots, m ;
$$

if $\lambda_{0}<0, \lambda_{1}<0$, then (2.9) holds if $p_{0}, p_{1}>m$.

We extend $A(t, s)$ and $B(t, s)$ with respect to $s$ outside $(0,1)$ by the zero value. Under conditions (2.3) we obtain continuous functions on $[0,1] \times \mathbf{R}$, see $(2.4)$. 


\section{Central Part Interpolation by Polynomials and Piece- wise Polynomials}

Given an interval $[a, b]$ and $m \in \mathbf{N}$, introduce the uniform grid consisting of $m$ points

$$
x_{i}=a+\left(i-\frac{1}{2}\right) h, \quad i=1, \ldots, m, h=\frac{b-a}{m} .
$$

Denote by $\Pi_{m}$ the Lagrange interpolation projection operator assigning to any $u \in C[a, b]$ the polynomial $\Pi_{m} u \in \mathcal{P}_{m-1}$ that interpolates $u$ at points (3.1); $\mathcal{P}_{m-1}$ is the set of polynomials of degree not exceeding $m-1$.

Lemma 7 [see [16]]. In the case of interpolation knots (3.1), for $f \in C^{m}[a, b]$ it holds

$$
\begin{gathered}
\max _{a \leq x \leq b}\left|f(x)-\left(\Pi_{m} f\right)(x)\right| \leq \theta_{m} h^{m} \max _{a \leq x \leq b}\left|f^{(m)}(x)\right|, \\
\theta_{m}=\frac{1 \cdot 3 \cdot \ldots \cdot(2 m-1)}{2 \cdot 4 \cdot \ldots \cdot 2 m} \cong(\pi m)^{-\frac{1}{2}},
\end{gathered}
$$

where $\theta_{m} \cong \epsilon_{m}$ means that $\theta_{m} / \epsilon_{m} \rightarrow 1$ as $m \rightarrow \infty$. Further, for $m=2 k$, $k \geq 1$,

$$
\begin{gathered}
\max _{x_{k} \leq x \leq x_{k+1}}\left|f(x)-\left(\Pi_{m} f\right)(x)\right| \leq \vartheta_{m} h^{m} \max _{a \leq x \leq b}\left|f^{(m)}(x)\right|, \\
\vartheta_{m}=2^{-2 m} \frac{m !}{((m / 2) !)} \cong \sqrt{2 / \pi} m^{-\frac{1}{2}} 2^{-m}
\end{gathered}
$$

whereas for $m=2 k+1, k \geq 1$,

$$
\begin{gathered}
\max _{x_{k} \leq x \leq x_{k+2}}\left|f(x)-\left(\Pi_{m} f\right)(x)\right| \leq \vartheta_{m} h^{m} \max _{a \leq x \leq b}\left|f^{(m)}(x)\right|, \\
\vartheta_{m}=\frac{2 \sqrt{3}}{9} \frac{(k !)^{2}}{(2 k+1) !} \cong \frac{2 \sqrt{3}}{9} \sqrt{2 / \pi} m^{-\frac{1}{2}} 2^{-m} .
\end{gathered}
$$

In the central parts of $[a, b]$, the interpolation process on the uniform grid has good stability properties as $m$ increases: in contrast to an exponential growth of $\left\|\Pi_{m}\right\|_{C[a, b] \rightarrow C[a, b]}$ as $m \rightarrow \infty$, it holds by the Runck's theorem (see [12])

$$
\left\|\Pi_{m}\right\|_{C[a, b] \rightarrow C\left[\frac{a+b}{2}-r h^{1 / 2}, \frac{a+b}{2}+r h^{1 / 2}\right]} \leq c_{r}(1+\log m),
$$

where the constant $c_{r}$ depends only on $r>0$.

Introduce in $\mathbf{R}$ the uniform grid $\{j h: j \in \mathbf{Z}\}$ where $h=1 / n, n \in \mathbf{N}$. Let $m \geq 2$ be fixed. Given a function $f \in C[-\delta, 1+\delta], \delta>0$, we define a piecewise polynomial interpolant $\Pi_{h, m} f \in C[0,1]$ as follows. On every subinterval $[j h,(j+1) h], 0 \leq j \leq n-1$, the function $\Pi_{h, m} f$ is defined independently from other subintervals as a polynomial $\Pi_{h, m}^{[j]} f \in \mathcal{P}_{m-1}$ of degree $\leq m-1$ by the conditions

$$
\Pi_{h, m}^{[j]} f(l h)=f(l h), \quad \text { for } l \in \mathbf{Z} \text { such that } l-j \in \mathbf{Z}_{m},
$$


where $\mathbf{Z}_{m}=\left\{k \in \mathbf{Z}:-\frac{m}{2}<k \leq \frac{m}{2}\right\}$. The $\Pi_{h, m} f$ is uniquely defined at interior knots and $\Pi_{h, m} f$ is continuous on $[0,1]$ (see [8]); the one side derivatives of the interpolant $\Pi_{h, m} f$ at the interior knots may be different.

Introduce the Lagrange fundamental polynomials $L_{k, m} \in \mathcal{P}_{m-1}, k \in \mathbf{Z}_{m}$ satisfying $L_{k, m}(l)=\delta_{k, l}$ for $l \in \mathbf{Z}_{m}$, where $\delta_{k, l}$ is the Kronecker symbol, $\delta_{k, l}=0$ for $k \neq l$ and $\delta_{k, k}=1$. An explicit formula for $L_{k, m}$ is given by

$$
L_{k, m}(t)=\prod_{l \in \mathbf{Z}_{m} \backslash\{k\}} \frac{t-l}{k-l}, \quad k \in \mathbf{Z}_{m} .
$$

Then (see [8])

$$
\left(\Pi_{h, m}^{[j]} f\right)(t)=\sum_{k \in \mathbf{Z}_{m}} f((j+k) h) L_{k, m}(n t-j), \quad j=0, \ldots, n-1 .
$$

For $m \geq 3, \Pi_{h, m} f$ uses values of $f$ outside of $[0,1]$. For $f \in C[0,1], \Pi_{h, m} f$ obtains a sense after an extension of $f$ onto $[-\delta, 1+\delta]$ with some $\delta>0$. We are in a lucky situation if $f \in C^{m}[0,1]$ satisfies the boundary conditions $f^{(j)}(0)=f^{(j)}(1)=0, j=1, \ldots, m$, cf. Lemma 6 and conditions $(2.9)$, then the simplest extension operator

$$
E_{\delta}: C[0,1] \rightarrow C[-\delta, 1+\delta], \quad\left(E_{\delta} f\right)(t)=\left\{\begin{array}{ll}
f(0), & -\delta \leq t \leq 0 \\
f(t), & 0 \leq t \leq 1 \\
f(1), & 1 \leq t \leq 1+\delta
\end{array}\right\}
$$

maintains the smoothness of $f$. The operator

$$
P_{h, m}:=\Pi_{h, m} E_{\delta}: C[0,1] \rightarrow C[0,1]
$$

is well defined and $P_{h, m}^{2}=P_{h, m}$, i.e., $P_{h, m}$ is a projector in $C[0,1]$. For $w_{h} \in \mathcal{R}\left(P_{h, m}\right)$ (the range of $\left.P_{h, m}\right)$ we have $w_{h}=P_{h, m} w_{h}=\Pi_{h, m} E_{\delta} w_{h}$, and due to $(3.8)$

$$
w_{h}(t)=\sum_{k \in \mathbf{Z}_{\mathbf{m}}}\left(E_{\delta} w_{h}\right)((j+k) h) L_{k, m}(n t-j),
$$

for $t \in[j h,(j+1) h], j=0, \ldots, n-1$, where $\left(E_{\delta} w_{h}\right)(i h)=w_{h}(i h)$ for $i=$ $0, \ldots, n,\left(E_{\delta} w_{h}\right)(i h)=w_{h}(0)$ for $i<0$ and $\left(E_{\delta} w_{h}\right)(i h)=w_{h}(1)$ for $i>n$. Thus $w_{h} \in \mathcal{R}\left(P_{h, m}\right)$ is uniquely determined on $[0,1]$ by its knot values $w_{h}(i h)$, $i=0, \ldots, n$. We conclude, that $\operatorname{dim} \mathcal{R}\left(P_{h, m}\right)=n+1$. It is also clear, that for a $w_{h} \in \mathcal{R}\left(P_{h, m}\right)$ we have $w_{h}=0$ if and only if $w_{h}(i h)=0, i=0, \ldots, n$.

On $[j h,(j+1) h]$ for $f \in C[-\delta, 1+\delta]$ the interpolant $\Pi_{h, m} f=\Pi_{h, m}^{[j]} f$ coincides with the polynomial interpolant $\Pi_{m} f$ constructed for $f$ on the interval $\left[a_{j}, b_{j}\right]$ where $a_{j}=\left(j-\frac{m-1}{2}\right) h, b_{j}=\left(j+\frac{m+1}{2}\right) h$ in the case of even $m$ and $a_{j}=\left(j-\frac{m}{2}\right) h, b_{j}=\left(j+\frac{m}{2}\right) h$ in the case of odd $m$; moreover, $[j h,(j+1) h]$ is a "central" part of $\left[a_{j}, b_{j}\right]$ on which the interpolation error can be estimated by (3.3) and (3.4).

In this way we obtain the following result. 
Lemma 8 [see [8]].

(i) For $f \in C^{m}[-\delta, 1+\delta]$,

$$
\max _{0 \leq t \leq 1}\left|f(t)-\left(\Pi_{h, m} f\right)(t)\right| \leq \vartheta_{m} h^{m} \max _{-\delta \leq t \leq 1+\delta}\left|f^{(m)}(t)\right|
$$

with $\vartheta_{m}$ defined in (3.3) and (3.4) respectively for even and odd $m$.

(ii) For $f \in V^{(m)}:=\left\{v \in C^{m}[0,1]: v^{(j)}(0)=v^{(j)}(1)=0, j=1, \ldots, m\right\}$, it holds

$$
\max _{0 \leq t \leq 1}\left|f(t)-\left(P_{h, m} f\right)(t)\right| \leq \vartheta_{m} h^{m} \max _{0 \leq t \leq 1}\left|f^{(m)}(t)\right|
$$

From (3.5) we obtain that

$$
\left\|P_{h, m}\right\|_{C[0,1] \rightarrow C[0,1]} \leq c(1+\log m) .
$$

Thus the norms $\left\|P_{h, m}\right\|_{C[0,1] \rightarrow C[0,1]}$ are uniformly bounded with respect to $n$. Together with (3.10), noticing that $V^{(m)}$ is dense in $C[0,1]$, the BanachSteinhaus theorem yields the following result.

Lemma 9. For any $f \in C[0,1], \max _{0 \leq t \leq 1}\left|f(t)-\left(P_{h, m} f\right)(t)\right| \rightarrow 0$, as $n \rightarrow \infty$.

\section{A Product Integration Method Based on the Central Part Interpolation}

We determine the approximate solution $v_{h}$ of equation (2.2) by solving the $n+1$ dimensional problem

$$
\begin{aligned}
v_{h}(t)= & \int_{0}^{1} \log |t-s| P_{h, m}\left(A(t, s) v_{h}(s)\right) d s \\
& +\int_{0}^{1} P_{h, m}\left(B(t, s) v_{h}(s)\right) d s+g(t), \quad 0 \leq t \leq 1 .
\end{aligned}
$$

Here $P_{h, m}$ (see (3.9)) is applied to the products $A(t, s) v_{h}(s)$ and $B(t, s) v_{h}(s)$ as functions of $s$, treating $t$ as a parameter. This is the operator form of a product interpolation method corresponding to the piecewise polynomial "central part" interpolation on the uniform grid $\{i h: i=0, \ldots, n\}$. Below we use the following notations for the integral operators of equations (1.1), (2.2) and (4.1) respectively:

$$
\begin{aligned}
& (T u)(x)=\int_{0}^{1}[a(x, y) \log |x-y|+b(x, y)] u(y) d y, \quad 0 \leq x \leq 1, \\
& (\mathcal{T} v)(t)=\int_{0}^{1}[A(t, s) \log |t-s|+B(t, s)] v(s) d s, \quad 0 \leq t \leq 1, \\
& \left(\mathcal{T}_{h} v\right)(t)=\int_{0}^{1}\left[\log |t-s| P_{h, m}(A(t, s) v(s))+P_{h, m}(B(t, s) v(s))\right] d s, \quad 0 \leq t \leq 1 .
\end{aligned}
$$




\section{Theorem 2.}

(i) Let $a$ and $b$ satisfy the conditions on Lemma $1, \mathcal{N}(I-T)=\{0\}, f \in$ $C[0,1]$ and let $p_{0}>1 /\left(1-\lambda_{0}\right)$ and $p_{1}>1 /\left(1-\lambda_{1}\right)$. Then for sufficiently large $n$, equation (4.1) has a unique solution $v_{h} \in C[0,1]$ and

$$
\left\|v-v_{h}\right\|_{\infty}:=\max _{t \in[0,1]}\left|v(t)-v_{h}(t)\right| \longrightarrow 0
$$

where $v \in C[0,1]$ is the unique solution of equation (2.2).

(ii) Let $a$ and $b$ satisfy the conditions of Lemma $2, \mathcal{N}(I-T)=\{0\}, f \in$ $C^{m, \lambda_{0}, \lambda_{1}}(0,1) ;$ let $p_{0}, p_{1}>m$ and let $p_{0}$ and $p_{1}$ satisfy the conditions $(2.5)$. Then

$$
\left\|v-v_{h}\right\|_{\infty} \leq c h^{m}\|f\|_{C^{m, \lambda_{0}, \lambda_{1}(0,1)}},
$$

constant $c$ in (4.3) is independent of $n$ and $f$.

Proof. (i) Accept the assumptions formulated in (i). We claim that $\mathcal{T}_{h} \rightarrow \mathcal{T}$ compactly in $C[0,1]$ as $n \rightarrow \infty$, i.e. (see $[1,15]$ )

$$
\left\|\mathcal{T}_{h} v-\mathcal{T} v\right\|_{\infty} \rightarrow 0 \text { for every } v \in C[0,1],
$$

$\left(v_{h}\right) \subset C[0,1],\left\|v_{h}\right\|_{\infty} \leq 1 \Rightarrow\left(\mathcal{T}_{h} v_{h}\right)$ is relatively compact in $C[0,1]$.

Indeed, the sets $\{A(t, \cdot): 0 \leq t \leq 1\}$ and $\{B(t, \cdot): 0 \leq t \leq 1\}$ are relatively compact in $C[-\delta, 1+\delta]$, and by Lemma 9 for a fixed $v \in C[0,1]$ extended by $v(s)=v(0)$ for $-\delta \leq s \leq 0$ and $v(s)=v(1)$ for $1 \leq s \leq 1+\delta$ it holds

$$
\begin{aligned}
& \sup _{0 \leq t \leq 1} \max _{0 \leq s \leq 1}\left|A(t, s) v(s)-P_{h, m}(A(t, s) v(s))\right| \rightarrow 0, \quad \text { as } n \rightarrow \infty, \\
& \sup _{0 \leq t \leq 1} \max _{0 \leq s \leq 1}\left|B(t, s) v(s)-P_{h, m}(B(t, s) v(s))\right| \rightarrow 0, \quad \text { as } n \rightarrow \infty .
\end{aligned}
$$

This together with the equality $\left\|P_{h, m}\right\| \leq c(1+\log m)$ (see (3.11)) implies (4.4). The proof of (4.5) can be built using the Arzela theorem.

Due to the condition $\mathcal{N}(I-T)=\{0\}$ also $\mathcal{N}(I-\mathcal{T})=\{0\}$. As well known (see $[1,2,5,15])$ relations $(4.4),(4.5)$ and $\mathcal{N}(I-\mathcal{T})=\{0\}$ imply that, for sufficiently large $n$ the operators $I-\mathcal{T}_{h}$ are invertible in $C[0,1]$ and the inverses are uniformly bounded:

$$
\left\|\left(I-\mathcal{T}_{h}\right)^{-1}\right\|_{C[0,1] \rightarrow C[0,1]} \leq c, \quad n \geq n_{0} .
$$

Let $v$ and $v_{h}$ be solutions of equations (2.2) and (4.1) respectively. Then $v-v_{h}=\left(I-\mathcal{T}_{h}\right)^{-1}\left(\mathcal{T} v-\mathcal{T}_{h} v\right)$ and

$$
\left\|v-v_{h}\right\|_{\infty} \leq c\left\|\mathcal{T} v-\mathcal{T}_{h} v\right\|_{\infty}, \quad n \geq n_{0},
$$

and, due to (4.4), the convergence (4.2) follows.

(ii) Let us prove the error estimate (4.3) under conditions (2.5) on $p_{0}$ and $p_{1}$, and $p_{0}, p_{1}>m$. For the solution $u$ of $(1.1)$ we have by the Theorem 1 
$u \in C^{m, \lambda_{0}, \lambda_{1}}(0,1)$. On the basis of $(2.7)-(2.9)$ and $(3.10)$ we find that

$$
\begin{aligned}
\left|(\mathcal{T} v)(t)-\left(\mathcal{T}_{h} v\right)(t)\right| & \mid \int_{0}^{1}[A(t, s) \log |t-s|+B(t, s)] v(s) d s \\
& -\int_{0}^{1}\left[\log |t-s| P_{h, m}(A(t, s) v(s))+P_{h, m}(B(t, s) v(s))\right] d s \mid \\
= & \left|\int_{0}^{1} \log \right| t-s \mid\left(I-P_{h, m}\right)(A(t, s) v(s)) d s+\int_{0}^{1}\left(I-P_{h, m}\right)(B(t, s) v(s) d s \mid \\
\leq & \int_{0}^{1}|\log | t-s\left|\left(I-P_{h, m}\right)(A(t, s) v(s))\right| d s+\int_{0}^{1} \mid\left(I-P_{h, m}\right)(B(t, s) v(s) \mid d s \\
\leq & c_{1} h^{m} \int_{0}^{1}|\log | t-s|| s^{-1+\delta_{0}}(1-s)^{-1+\delta_{1}} d s \cdot\|u\|_{C^{m, \lambda_{0}, \lambda_{1}}(0,1)} \\
& +c_{2} h^{m} \int_{0}^{1} s^{-1+\delta_{0}}(1-s)^{-1+\delta_{1}}|\log s(1-s)| d s \cdot\|u\|_{C^{m, \lambda_{0}, \lambda_{1}}(0,1)} \\
\leq & c h^{m}\|u\|_{C^{m, \lambda_{0}, \lambda_{1}}(0,1)} \cdot
\end{aligned}
$$

This proves (4.3) and completes the proof of the Theorem.

Let us derive the matrix form of the product interpolation method (4.1). From the definition of the operator $P_{h, m}$ (see (3.8)):

$$
\begin{aligned}
v_{h}(t)= & \sum_{j=0}^{n-1} \int_{j h}^{(j+1) h} \log |t-s| \sum_{k \in \mathbf{Z}_{m}} A(t,(j+k) h) v_{h}((j+k) h) L_{k, m}(n s-j) d s \\
& +\sum_{j=0}^{n-1} \int_{j h}^{(j+1) h} \sum_{k \in \mathbf{Z}_{m}} B(t,(j+k) h) v_{h}((j+k) h) L_{k, m}(n s-j) d s \\
& +g(t), \quad 0 \leq t \leq 1 .
\end{aligned}
$$

We obtain the algebraic system of linear equations with respect to the grid values $v_{h}(i h), i=0, \ldots, n$ by collocating at the points $i h$ :

$$
\begin{aligned}
v_{h}(i h)= & \sum_{j=0}^{n-1} \sum_{k \in \mathbf{Z}_{m}}\left\{A(i h,(j+k) h) \int_{j h}^{(j+1) h} \log |i h-s| L_{k, m}(n s-j) d s\right. \\
& \left.+B(i h,(j+k) h) \int_{j h}^{(j+1) h} L_{k, m}(n s-j) d s\right\} v_{h}((j+k) h) \\
& +g(i h), \quad i=0, \ldots, n
\end{aligned}
$$

note that $A(i h,(j+k) h)=0$ and $B(i h,(j+k) h)=0$ for $j+k \leq 0$ and for $j+k \geq n$, thus in the r.h.s the values $v_{h}(l h)$ with $l \leq 0$ and $l \geq n$ actually are not exploited. 
With the change of variables $n s-j=\sigma$ we see that

$$
\begin{aligned}
& \int_{j h}^{(j+1) h} \log |i h-s| L_{k, m}(n s-j) d s \\
& \quad=h\left[\log h \int_{0}^{1} L_{k, m}(\sigma) d \sigma+\int_{0}^{1} \log |i-j-\sigma| L_{k, m}(\sigma) d \sigma\right]
\end{aligned}
$$

and

$$
\int_{j h}^{(j+1) h} L_{k, m}(n s-j) d s=h \int_{0}^{1} L_{k, m}(\sigma) d \sigma, \quad j=0, \ldots, n-1, k \in \mathbf{Z}_{m},
$$

so we have to compute integrals

$$
\begin{aligned}
\alpha_{i^{\prime}, k} & :=\log h \int_{0}^{1} L_{k, m}(\sigma) d \sigma+\int_{0}^{1} \log \left|i^{\prime}-\sigma\right| L_{k, m}(\sigma) d \sigma \\
i^{\prime} & =-n+1, \ldots, n, k \in \mathbf{Z}_{m}
\end{aligned}
$$

and

$$
\beta_{k}:=\int_{0}^{1} L_{k, m}(\sigma) d \sigma, \quad k \in \mathbf{Z}_{m}
$$

The system takes the form:

$$
\begin{aligned}
v_{h}(i h)= & h \sum_{j=0}^{n-1} \sum_{k \in \mathbf{Z}_{m}}\left\{A(i h,(j+k) h) \alpha_{i-j, k}+B(i h,(j+k) h) \beta_{k}\right\} v_{h}((j+k) h) \\
& +g(i h),
\end{aligned}
$$

$i=0, \ldots, n$, or collecting in the r.h.s. the coefficients by $v_{h}((j+k) h)$ with fixed $j+k=l$,

$$
v_{h}(i h)=\sum_{l=1}^{n-1} c_{i, l} v_{h}(l h)+g(i h), \quad i=0, \ldots, n,
$$

where

$$
\begin{aligned}
c_{i, l} & =h\left[A(i h, l h) \sum_{\left\{k \in \mathbf{Z}_{m}: 0 \leq l-k \leq n-1\right\}} \alpha_{i-l+k, k}+B(i h, l h) \sum_{k \in \mathbf{Z}_{m}} \beta_{k}\right], \\
i & =0, \ldots, n, l=1, \ldots, n-1 .
\end{aligned}
$$

We took into account that $A(i h, l h)=0$ and $B(i h, l h)=0$ for $l \leq 0$ and $l \geq n$. Having determined $v_{h}(i h), i=0, \ldots, n$, through solving the system (4.6), the collocation solution $v_{h}(t)$ at any intermediate point $t \in[j h,(j+1) h], j=$ $0, \ldots, n-1$, is given by

$$
v_{h}(t)=\sum_{k \in \mathbf{Z}_{m}}\left\{\begin{array}{ll}
v_{h}(0), & j+k \leq 0 \\
v_{h}((j+k) h), & 1 \leq j+k \leq n-1 \\
v_{h}(1), & j+k \geq n
\end{array}\right\} \cdot L_{k, m}(n t-j),
$$

where $L_{k, m}$ is the Lagrange fundamental polynomial defined in (3.7). 


\section{Numerical Example}

For testing the algorithm (4.6) we consider equation (1.1) with $b(x, y) \equiv 0$, $a(x, y)=y^{-\lambda_{0}}(1-y)^{-\lambda_{1}}, \lambda_{0}<1, \lambda_{1}<1$ :

$$
u(x)=\int_{0}^{1} y^{-\lambda_{0}}(1-y)^{-\lambda_{1}} \log |x-y| d y+f(x), \quad 0 \leq x \leq 1 .
$$

Table 1. $m=2, p=9, \lambda_{0}=\lambda_{1}=0.75$.

Table 2. $m=3, p=12, \lambda_{0}=\lambda_{1}=0.75$.

\begin{tabular}{rll}
\hline$n$ & $\epsilon_{2, n, 9}$ & $\frac{\epsilon_{2, n, 9}}{\epsilon_{2,2 n, 9}}$ \\
\hline 4 & $3.26 \mathrm{E}-02$ & \\
8 & $1.84 \mathrm{E}-02$ & 1.77 \\
16 & $5.77 \mathrm{E}-03$ & 3.19 \\
32 & $1.48 \mathrm{E}-03$ & 3.89 \\
64 & $3.69 \mathrm{E}-04$ & 4.01 \\
128 & $9.19 \mathrm{E}-05$ & 4.02 \\
256 & $2.29 \mathrm{E}-05$ & 4.01 \\
512 & $5.71 \mathrm{E}-06$ & 4.01 \\
1024 & $1.42 \mathrm{E}-06$ & 4.02 \\
\hline
\end{tabular}

\begin{tabular}{rll}
\hline$n$ & $\epsilon_{3, n, 12}$ & $\frac{\epsilon_{3, n, 12}}{\epsilon_{3,2 n, 12}}$ \\
\hline 4 & 0.17 & \\
8 & $3.11 \mathrm{E}-02$ & 5.47 \\
16 & $4.39 \mathrm{E}-03$ & 7.08 \\
32 & $5.42 \mathrm{E}-04$ & 8.10 \\
64 & $6.72 \mathrm{E}-05$ & 8.07 \\
128 & $8.37 \mathrm{E}-06$ & 8.03 \\
256 & $1.04 \mathrm{E}-06$ & 8.05 \\
512 & $1.30 \mathrm{E}-07$ & 8 \\
1024 & $1.62 \mathrm{E}-08$ & 8.02 \\
\hline
\end{tabular}

Table 3. $m=4, p=17, \lambda_{0}=\lambda_{1}=0.75$.

Table 4. $m=5, p=21, \lambda_{0}=\lambda_{1}=0.75$.

\begin{tabular}{rlc}
\hline$n$ & $\epsilon_{4, n, 9}$ & $\frac{\epsilon_{4, n, 17}}{\epsilon_{4,2 n, 17}}$ \\
\hline 4 & 0.14 & \\
8 & $1.42 \mathrm{E}-02$ & 9.86 \\
16 & $2.55 \mathrm{E}-03$ & 5.92 \\
32 & $2.13 \mathrm{E}-04$ & 11.51 \\
64 & $1.43 \mathrm{E}-05$ & 14.76 \\
128 & $9.06 \mathrm{E}-07$ & 15.78 \\
256 & $5.66 \mathrm{E}-08$ & 16.01 \\
512 & $3.53 \mathrm{E}-09$ & 16.03 \\
1024 & $2.18 \mathrm{E}-10$ & 16.19 \\
\hline
\end{tabular}

\begin{tabular}{rlc}
\hline$n$ & $\epsilon_{5, n, 21}$ & $\frac{\epsilon_{5, n, 21}}{\epsilon_{5,2 n, 21}}$ \\
\hline 4 & 0.20 & \\
8 & $2.39 \mathrm{E}-02$ & 8.37 \\
16 & $3.46 \mathrm{E}-03$ & 6.91 \\
32 & $1.27 \mathrm{E}-04$ & 27.24 \\
64 & $4.53 \mathrm{E}-06$ & 28.04 \\
128 & $1.43 \mathrm{E}-07$ & 31.68 \\
256 & $4.46 \mathrm{E}-09$ & 32.06 \\
512 & $1.43 \mathrm{E}-10$ & 31.19 \\
1024 & $6.52 \mathrm{E}-12$ & 21.93 \\
\hline
\end{tabular}

We set $u(x)=x^{\lambda_{0}}(1-x)^{\lambda_{1}}, \lambda_{0}<1, \lambda_{1}<1$ to be the exact solution; it corresponds to $f(x)=x^{\lambda_{0}}(1-x)^{\lambda_{1}}-x \log x-(1-x) \log (1-x)+1,0<x<1$. We composed system (4.6) for $m=2,3,4,5, n=2^{k}$ with $k=1,2, \ldots, 10$. In functions $a$ and $f$ we used various values of $\lambda_{0}$ and $\lambda_{1}$; in the change of variables (2.1) we used different values of the smoothing parameter $\max \left\{p_{0}, p_{1}\right\}=p \in \mathbf{N}$ depending on $\lambda_{0}$ and $\lambda_{1}$ and satisfying the conditions (2.5). In Tables 1-8 the errors

$$
\epsilon_{m, n, p}:=\max _{0 \leq i \leq n}\left|v(i h)-v_{h}(i h)\right|
$$

and the quotients $\epsilon_{m, n, p} / \epsilon_{m, 2 n, p}$ are presented. The expected limit value of $\epsilon_{m, n, p} / \epsilon_{m, 2 n, p}$ is $2^{m}$. As results in the tables show, the numerical results confirm our theoretical results (see (4.3)) quite well. 
Table 5. $m=2, p=9, \lambda_{0}=\lambda_{1}=0.25$.

\begin{tabular}{rll}
\hline$n$ & $\epsilon_{2, n, 9}$ & $\frac{\epsilon_{2, n, 9}}{\epsilon_{2,2 n, 9}}$ \\
\hline 4 & 0.10 & \\
8 & $4.32 \mathrm{E}-02$ & 2.31 \\
16 & $1.17 \mathrm{E}-02$ & 3.69 \\
32 & $2.99 \mathrm{E}-03$ & 3.91 \\
64 & $7.55 \mathrm{E}-04$ & 3.96 \\
128 & $1.90 \mathrm{E}-04$ & 3.97 \\
256 & $4.77 \mathrm{E}-05$ & 3.98 \\
512 & $1.19 \mathrm{E}-05$ & 4.01 \\
1024 & $2.99 \mathrm{E}-06$ & 3.98 \\
\hline
\end{tabular}

Table 6. $m=3, p=15, \lambda_{0}=\lambda_{1}=0.25$.

\begin{tabular}{rll}
\hline$n$ & $\epsilon_{3, n, 15}$ & $\frac{\epsilon_{3, n, 15}}{\epsilon_{3,2 n, 15}}$ \\
\hline 4 & 0.16 & \\
8 & $3.11 \mathrm{E}-02$ & 5.14 \\
16 & $6.01 \mathrm{E}-03$ & 5.10 \\
32 & $8.56 \mathrm{E}-04$ & 7.13 \\
64 & $1.11 \mathrm{E}-04$ & 7.71 \\
128 & $1.40 \mathrm{E}-05$ & 7.93 \\
256 & $1.76 \mathrm{E}-06$ & 7.95 \\
512 & $2.21 \mathrm{E}-07$ & 7.96 \\
1024 & $2.76 \mathrm{E}-08$ & 8.01 \\
\hline
\end{tabular}

Table 7. $m=2, p=21, \lambda_{0}=0.2, \lambda_{1}=0.9$.

\begin{tabular}{rll}
\hline$n$ & $\epsilon_{2, n, 21}$ & $\frac{\epsilon_{2, n, 21}}{\epsilon_{2,2 n, 21}}$ \\
\hline 4 & 0.24 & \\
8 & $8.51 \mathrm{E}-02$ & 5.67 \\
16 & $2.32 \mathrm{E}-02$ & 2.82 \\
32 & $6.18 \mathrm{E}-03$ & 3.67 \\
64 & $1.59 \mathrm{E}-03$ & 3.89 \\
128 & $4.06 \mathrm{E}-04$ & 3.92 \\
256 & $1.02 \mathrm{E}-04$ & 3.98 \\
512 & $2.56 \mathrm{E}-05$ & 3.98 \\
1024 & $6.40 \mathrm{E}-06$ & 4 \\
\hline
\end{tabular}

Table 8. $m=3, p=31, \lambda_{0}=0.2, \lambda_{1}=0.9$.

\begin{tabular}{rll}
\hline$n$ & $\epsilon_{3, n, 31}$ & $\frac{\epsilon_{3, n, 31}}{\epsilon_{3,2 n, 31}}$ \\
\hline 4 & 0.35 & \\
8 & 0.12 & 4.91 \\
16 & $2.29 \mathrm{E}-02$ & 2.92 \\
32 & $2.92 \mathrm{E}-03$ & 7.84 \\
64 & $3.82 \mathrm{E}-04$ & 7.64 \\
128 & $4.87 \mathrm{E}-05$ & 7.84 \\
256 & $6.17 \mathrm{E}-06$ & 7.89 \\
512 & $7.75 \mathrm{E}-07$ & 7.96 \\
1024 & $9.71 \mathrm{E}-08$ & 7.98
\end{tabular}

\section{Acknowledgment}

This work was supported by the Estonian Science Foundation grant 9104.

The author would like to thank Gennadi Vainikko and Arvet Pedas for valuable discussion and remarks regarding the manuscript.

\section{References}

[1] P.M. Anselone. Collectively Compact Operator Approximation Theory. Prentice Hall, New Jersey, 1971.

[2] K.E. Atkinson. The Numerical Solution of Integral Equations of the Second Kind. Cambridge University Press, Cambridge, 1997.

[3] M. Kolk and A. Pedas. Numerical solution of Volterra integral equations with weakly singular kernels which may have a boundary singularity. Math. Model. Anal., 14(1):79-89, 2009. http://dx.doi.org/10.3846/1392-6292.2009.14.79-89.

[4] M. Kolk, A. Pedas and G. Vainikko. High-order methods for Volterra integral equations with general weak singularities. Numer. Funct. Anal. Optim., 30(910):1002-10024, 2009. http://dx.doi.org/10.1080/01630560903393154.

[5] R. Kress. Linear Integral Equations. Springer, Berlin, 1989. 
[6] G. Monegato and L. Scuderi. High order methods for weakly singular integral equations with nonsmooth input functions. Math. Comp., 67:1493-1515, 1998. http://dx.doi.org/10.1090/S0025-5718-98-01005-9.

[7] K. Orav-Puurand, A. Pedas and G. Vainikko. Nystroem type methods for Fredholm integral equations with weak singularities. J. Comput. Appl. Math., 234(9):2848-2858, 2010. http://dx.doi.org/10.1016/j.cam.2010.01.033.

[8] K. Orav-Puurand and G. Vainikko. Central part interpolation schemes for integral equations. Numer. Funct. Anal. Optim., 30(3-4):352-370, 2009. http://dx.doi.org/10.1080/01630560902841153.

[9] A. Pedas. Nystroem type methods for a class of logarithmic singular Fredholm integral equations. In Theodore E. Simos, George Psihoyios, Ch. Tsitouras and Zacharias Anastassi(Eds.), International Conference on Numerical Analysis and Applied Mathematics, volume 1389, pp. 477-480, Halkidiki, Greece, 2011. Amer. Inst. Physics.

[10] A. Pedas and G. Vainikko. Smoothing transformation and piecewise polynomial collocation for weakly singular integral equations. Computing, 73:271-293, 2004. http://dx.doi.org/10.1007/s00607-004-0088-9.

[11] A. Pedas and G. Vainikko. Integral equations with diagonal and boundary singularities of the kernel. J. Anal. Appl. (ZAA), 25:487-516, 2006.

[12] P. Runck. Über Konvergenzfragen bei Polynominterpolation mit equidistanten Knoten I, II. J. Reine Angew. Math., 208; 210:51-69; 175-204, 1961; 1962.

[13] E. Vainikko and G. Vainikko. A spline product quasi-interpolation method for weakly singular Fredholm integral equations. SIAM J. Numer. Anal., 46(4):1799-1820, 2008. http://dx.doi.org/10.1137/070693308.

[14] E. Vainikko and G. Vainikko. Product quasi-interpolation in logarithmically singular integral equations. Math. Model. Anal., 17(5):696-714, 2012. http://dx.doi.org/10.3846/13926292.2012.736089.

[15] G. Vainikko. Funktionalanalysis der Diskretisierungsmethoden. Teubner Verlag, Leipzig, 1976.

[16] G. Vainikko. Weakly singular integral equations. Lecture notes, University of Tartu, Helsinki University of Technology, available from Internet: http://www.ut.ee/ gen/WSIElecturesSIAM.pdf, 2006-2007. 